\title{
Paragangliomas of the Head and Neck
}

\author{
A. Cemal Ümit Işı ${ }^{a}$ Mehmet İmamoğlu ${ }^{a}$ Cihangir Erem ${ }^{b}$ Ahmet Sarı ${ }^{c}$ \\ Departments of ${ }^{\mathrm{a}}$ Otolaryngology, ${ }^{\mathrm{b}}$ Internal Medicine, Division of Endocrinology and Metabolism, and \\ 'Radiology, Faculty of Medicine, Karadeniz Technique University, Trabzon, Turkey
}

\section{Key Words}

Carotid body tumor $\cdot$ Glomus tumor $\cdot$ Paraganglioma •

Vagus nerve

\begin{abstract}
Objectives: The purpose of this study was to determine the clinical characteristics of carotid body tumors and to analyze the benefits of different treatment modalities. Subjects and Methods: The clinical records of 13 patients with paragangliomas treated during the period of 1995-2005 were reviewed for age at diagnosis, sex, initial symptoms, duration of symptoms, evidence of secretory function, family history, radiographic studies, pre- and posttreatment cranial nerve deficits, multicentric tumors, angiography, operative procedures and findings, and complications. The diagnoses were established by computerized tomography and magnetic resonance imaging scanning. Angiography was performed on 3 patients with carotid body tumor. Results: All of the patients were followed up for a duration ranging from 16 months to 9 years. The median follow-up was 5 years. Of all 18 head and neck tumors confirmed in 13 patients, 16 were in the carotid body, 1 in the vagus nerve and 1 in the middle ear cleft. Four patients had multiple paragangliomas, 2 bilateral synchronous and 1 bilateral asynchronous paragangliomas. The remaining patient had 3 asynchronous paragangliomas, i.e. left jugulotympanic, right carotid body and left vagal paraganglioma. All patients underwent successful surgical resection of the tumor after appropriate preoperative
\end{abstract}

(c) 2007 S. Karger AG, Basel

1011-7571/07/0163-0209\$23.50/0

Fax +41613061234

E-Mail karger@karger.ch

www.karger.com
Accessible online at: www.karger.com/mpp investigation. Conclusion: This study shows that surgical treatment is acceptably safe and effective in treating these neoplasms.

Copyright $\odot 2007$ S. Karger AG, Basel

\section{Introduction}

Head and neck paragangliomas or glomus tumors are non-chromaffin tumors that originate from paraganglionic cells migrating from the neural crest in close relation with autonomic ganglion cells [1]. Earlier literature referred to these tumors as chemodectoma, but the more accurate term 'paraganglioma' is preferred. Multiple paragangliomas and bilaterality have been associated with a positive family history and recurrence. Malignancy in paragangliomas is more common in the nonfamilial than in the hereditary form [2]. In the head and neck, they commonly arise from the carotid body, tympanic plexus, jugular bulb, vagal and sympathetic ganglia [3]. The most common site of origin of head and neck paragangliomas is at the carotid bifurcation, followed by the region of the ganglion nodosum. Carotid paragangliomas (CPGs) are usually present as a neck mass [4]. Enlarging tumors may extend along the carotid artery involving adjacent cranial nerves, including the vagus, hypoglossal nerves and the sympathetic chain. Typically, tympanic paragangliomas are seen with pulsatile tinnitus and hearing loss and may extend into the jugular 


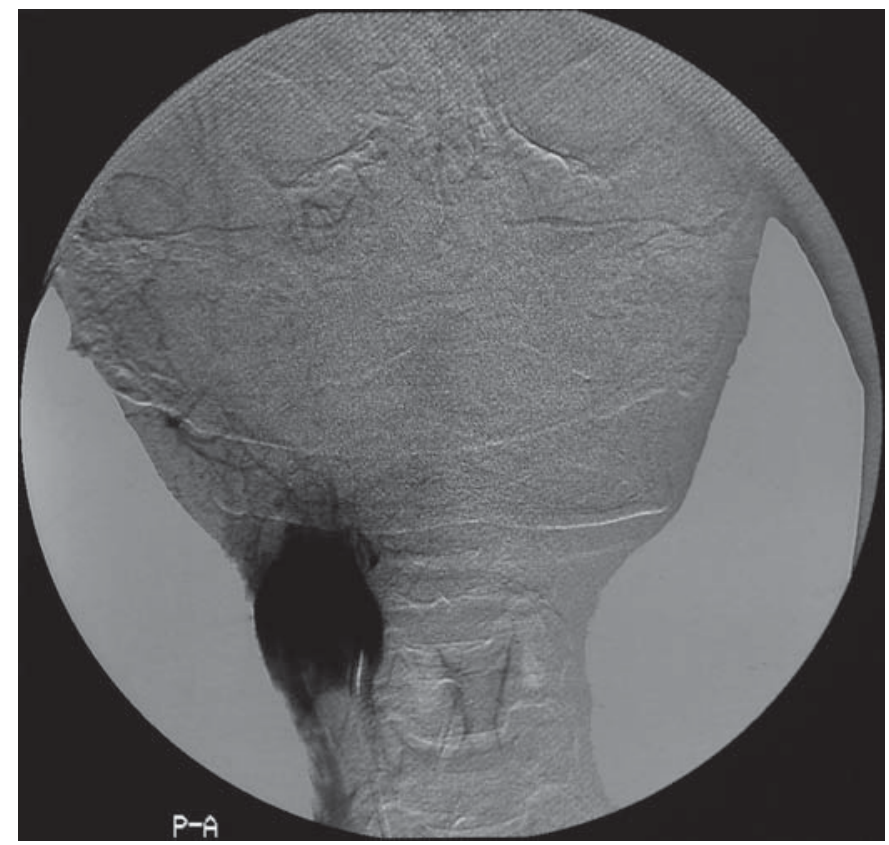

Fig. 1. Digital subtraction angiography demonstrates a hypervascularized mass with splaying of the external and internal carotid artery, occupying and deforming the carotid bifurcation.

bulb. The purpose of this article was to review the experience of our institution with paragangliomas. In addition, clinical characteristics of the tumor and diagnostic and therapeutic approaches to this rare disease are discussed.

\section{Subjects and Methods}

The records of patients who underwent resection of paragangliomas in the Faculty of Medicine at Karadeniz Technique University between 1995 and 2005 were examined. The clinical records of each patient were reviewed for age at diagnosis, sex, initial symptoms, duration of symptoms, evidence of secretory function, family history, radiographic studies, pre- and posttreatment cranial nerve deficits, multicentric tumors, angiography, operative procedures and findings, and complications. Eighteen tumors in 13 patients were identified and confirmed histologically as paragangliomas. Tachycardia, hypertension and tremors could also indicate possible endocrine activity. Every patient with a paraganglioma was screened for catecholamine-secreting tumors by urinary/plasma catecholamine determinations.

While undergoing neck examination, it was found that two asymptomatic patients had lesions; one of these was discovered during the follow-up evaluation of the contralateral neck 2 years after excision of a carotid body tumor at another institution, and the other during a routine evaluation for a family history of carotid body tumor. The duration of neck mass for patients ranged

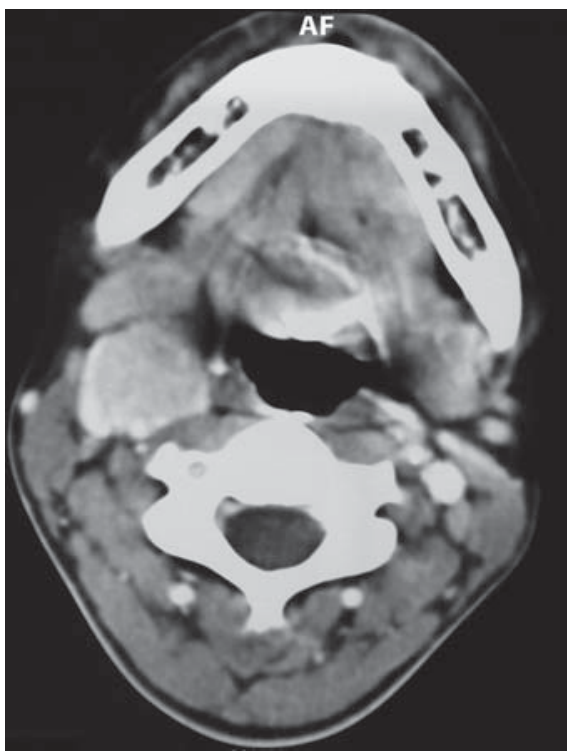

Fig. 2. Axial CT scan reveals a carotid body tumor at the bifurcation of the right carotid artery. $\mathrm{AF}=$ Anterior fossa. from 1 to 12 years. All patients underwent complete head and neck examination and digital subtraction carotid angiography (fig. 1, 2). Based on this information, the tumors were classified according to Shamblin et al. [5]: type I, small tumors with minimal arterial attachment; type II, usually larger, exhibiting moderate arterial attachment and partially surrounding both carotid arteries; type III, characterized by encasement of the carotid bifurcation. Computerized tomography (CT) or magnetic resonance imaging (MRI) was used preoperatively to evaluate possible tumor invasion.

$\mathrm{A}^{123}$ I-metaiodobenzylguanidine (MIBG) scan was used preoperatively to identify possible multicentric paragangliomas in patients with family histories. Vocal cord function was recorded before and after the operation by laryngoscopic examination.

The operation for a CPG required an oblique incision along the anterior border of the sternocleidomastoid muscle. The carotid sheath was then opened with identification of the common internal carotid artery, external carotid arteries and internal jugular vein, as well as the vagus and hypoglossal nerves preserved by vessel loops (fig. 3). Dissection in the subadventitial tissue plane was performed to safely separate the lesion from the artery by sharp dissection in all patients. In CPG, the dissection from the internal carotid artery was usually started posterolaterally because this was found to be the area least affected by the tumor. The dissection was carefully extended distally to the bifurcation of the carotid artery where the caudal borders of the tumor originate. After ligating the ascending pharyngeal artery which is often met at the medial surface of the external carotid artery, the tumor was retracted superiorly. If the tumor was small, simple 
Fig. 3. Intraoperative view of the carotid body tumor. The common carotid artery (CCA), the internal carotid artery (ICA), the internal jugular vein (IJV), the carotid paraganglioma (CPG), the hypoglossal $(\mathrm{H})$ and the vagus nerves $(\mathrm{V})$ can be observed.

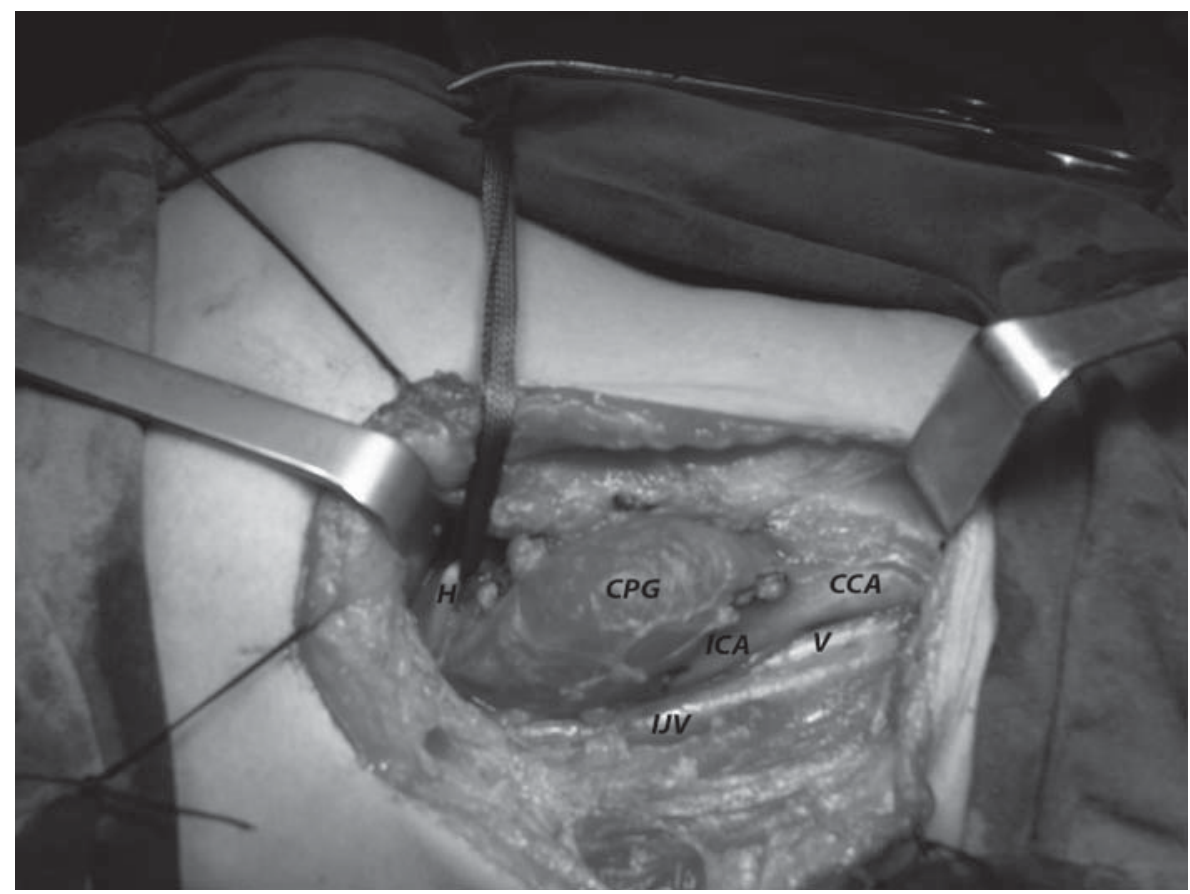

excision was performed; however, if there was extensive arterial invasion, the external carotid artery was sacrificed to avoid excessive bleeding and mobilize the tumor posteriorly from the internal carotid artery. Simple arterial laceration required primaryrepair after the temporary application of atraumatic vascular clamps.

\section{Results}

The 13 patients with a median age of 32 years (range 25-48) underwent surgery and were followed up for a duration ranging from 16 months to 9 years. The median follow-up was 5 years. The median duration between the onset of symptoms and diagnosis of carotid body paraganglioma was 2 years (range 1-12). In 4 patients, the tumor was familial; these were usually bilateral or combined with jugulotympanic or vagal paraganglioma (VP).

All 13 patients had 16 carotid body tumors, either alone or together with pheochromocytoma, jugulotympanic paraganglioma or VP. One patient with a jugulotympanic paraganglioma had aural fullness and hearing loss, but did not present with cranial nerve palsies. The carotid body tumors presented as neck masses in 16, dysphagia in 1 , oropharyngeal mass in 1 and Horner's syndrome in 1 patient. While undergoing a neck dissection for a metastatic lymph node, 1 patient with laryngeal cancer was found to have a carotid body tumor intraoperatively.

Eleven patients with CPGs exhibited neck mass at the level of the carotid bifurcation fixed in the vertical plane, but horizontally mobile. Multiple paragangliomas were found in 4 patients: 2 patients had bilateral synchronous tumors and 2 bilateral asynchronous paraganglioma. One patient with laryngeal carcinoma also had a CPG in the contralateral neck, and another with bilateral CPGs was found to have multinodular goiter (table 1). Nonparoxysmal hypertension was found in 3 patients and during further evaluation, and 2 developed adrenal pheochromocytoma. In addition, in a patient with asynchronous left jugulotympanic paraganglioma, right CPG and left $\mathrm{VP}$, the interval between the first and the last paraganglioma was 14 years. This patient had subclinical hypothyroidism due to Hashimoto's thyroiditis. Three patients underwent angiography, and the ascending pharyngeal artery represented the major blood supply for all tumors. A ${ }^{131}$ I-MIBG scan revealed uptake within the adrenal mass in 2 patients. The 3 patients with CPG displayed highly vascularized masses within the carotid bifurcations with their typical broadening. Three patients underwent therapeutic embolization before surgery with reduction in vascularity. One patient with VP showed a 
Table 1. Presentation of patients with paragangliomas

\begin{tabular}{lllll}
\hline $\begin{array}{l}\text { Age } \\
\text { years }\end{array}$ & Gender & Site & Symptoms & Other disease \\
\hline 36 & female & right carotid & $\begin{array}{l}\text { neck mass } \\
\text { deficits }\end{array}$ \\
42 & male & left carotid & dysphagia & no \\
37 & male & right carotid & neck mass & no \\
48 & male & left carotid & neck mass, hoarseness & laryngeal carcinoma \\
35 & male & right carotid & neck mass & no \\
39 & male & left carotid & neck mass & no \\
25 & male & right carotid & neck mass & no \\
43 & female & right carotid, left vagal & neck mass, hearing loss, hypertension & Hashimoto's thyroiditis \\
& & and jugulotympanic & neck mass & no \\
52 & male & left carotid & neck mass, hypertension, tachycardia & pheochromocytoma, EMNG \\
46 & male & right and left carotid & neck mass, hypertension, tachycardia & pheochromocytoma, EUNG \\
38 & female & right and left carotid & no \\
45 & male & right carotid & Horner's syndrome & EMNG \\
47 & female & right and left carotid & neck mass & X, XII (S) \\
\hline
\end{tabular}

No metastasis or functional status was present in any of these tumors. EMNG = Euthyroid multinodular goiter; EUNG = euthyroid unnodular goiter; $\mathrm{S}=$ sacrificed.

highly vascular mass medial to the mandible, displacing the carotid vessels anteriorly. Fourteen tumors were classified at the time of operation by the attending surgeon according to the classification system of Shamblin et al. [5], which is based on the size and difficulty of resection. Of the 14 carotid body tumors identified, 4 were of type I, 7 of type II and 3 of type III. Nerve deficits comprised hypoglossal nerve paralysis in 1 patient with CPG and vagal paralysis in patients with VP. The sympathetic chain was sacrificed in 1 patient with CPG. One patient was referred with the complication of an injury to the vagus and hypoglossal nerves after operation in another center. A minor lesion of the arterial wall was repaired in 3 patients. Patch angioplasty or a polytetrafluoroethylene graft was not used for vascular reconstruction. Three ligations of the external carotid artery were required for access to large tumors. There were no hospital deaths or wound infections in this series.

\section{Discussion}

Head and neck paragangliomas are recognized to occur in two forms: sporadic and familial forms [6]. It is estimated that the familial incidence of head and neck paragangliomas is approximately $5 \%$. If such a history is present, there is a $78-87 \%$ possibility of multiple tumors [7]. Four patients in our series had a family history of paraganglioma. These patients with the familial form had bilateral CPGs. The most common types, in order of decreasing frequency, are carotid body tumor, jugulotympanic tumor and vagal body tumor. In jugulotympanic paraganglioma, the clinical course consists of slow continuous growth with few symptoms such as pulsatile tinnitus, aural fullness or hearing loss. Cranial nerve deficits may be seen with larger tumors. The eighth patient with jugulotympanic paraganglioma had aural fullness and hearing loss but did not present with cranial nerve palsies.

A carotid body tumor usually presents as neck mass, headache or neck pain in $35 \%$, dizziness in $8 \%$, hoarseness in $8 \%$ and dysphagia in $8 \%$ of patients [8]. The involvement of the cranial nerves, especially the vagus and the hypoglossal, occurs in $10 \%$ of the cases. These slowgrowing tumors are mostly benign; however, malignant transformation may occur in up to $10 \%$ of cases. Metastases are found in less than 5\% and multiple tumors in 5\% of cases [9]. In our study, there was no malignant paraganglioma, but 1 patient with CPG also had a squamous cell laryngeal carcinoma. During ipsilateral neck dissection in a patient with transglottic laryngeal cancer, a contralateral atypical neck mass was identified and neck dissection performed. This mass was unexpectedly confirmed as a carotid body tumor.

The patients with CPG displayed highly vascularized masses within the carotid bifurcations with their typical 
broadening. In this series, a bilateral carotid body tumor was found during a familial MRI screening on the neck.

CT and MRI have been advocated to establish the diagnosis by demonstrating the vascular nature of the tumor, but cannot replace angiography in evaluating the arterial supply [10]. A CT scan can suggest the diagnosis and is useful to evaluate high lesions extending to the base of the skull and into the retropharyngeal area. Commonly, patients are initially examined with MRI; multiplanar imaging capability with a high soft-tissue contrast technique may soon make MRI the primary diagnostic tool when carotid body tumor is suspected [6]. In this series, a bilateral carotid body tumor was found during a familial MRI screening on the neck.

This provides the surgeon with the advantage of locating displaced vessels and also gives an enhanced view for the identification of those feeding vessels to be ligated. Thus, more unpredictable and less accessible feeding vessels from the internal carotid supply would be identified and appropriately managed $[11,12]$. Preoperative endocrine studies (such as urinary metanephrine, normetanephrine, norepinephrine, epinephrine, vanillylmandelic acid, homovanillic acid and dopamine) should be obtained in patients with tachycardia, flushing, headaches, pallor, tremor, nausea or hypertension. Patients with multiple and/or familial instances of paragangliomas are frequently found to have catecholamine-secreting tumors [13].

Three patients in the present study had a history of hypertension. After carotid body tumor resection, the patients continued to exhibit high blood pressure postoperatively, suggesting that the hypertension was not related to the carotid body tumors. In fact, during further evaluation, adrenal pheochromocytoma was found in 2 cases having paroxysmal hypertension associated with severe headaches, palpitation, nervousness and sweating. In 2 patients, there were no clinical and laboratory evidence, including hypercalcemia, an increased parathyroid hormone level, an increased serum calcitonin level, a marfanoid habitus, multiple mucosal neuromas, retinal and cerebellar hemangioblastomas, renal cell carcinoma, as well as renal and pancreatic cysts. Furthermore, in 2 patients, there were a variety of skin manifestations, including café-au-lait spots, subcutaneous multiple neurofibromas and axillary and inguinal freckles, as well as neural gliomas (e.g., optic nerve) and hamartomas of the iris (Lisch nodules), to suggest other familial syndromes, e.g., multiple endocrine neoplasia type 2 , von Hippel-Lindau disease and neurofibromatosis type 1 [14].

Paragangliomas of the Head and Neck
Recent studies suggest that germline mutations are an important cause of familial and isolated pheochromocytoma. While the combination of familial carotid body tumors and pheochromocytoma is rare, a patient who remains hypertensive after removal of a carotid body tumor should be evaluated carefully to exclude pheochromocytoma. This fact is demonstrated by the 2 siblings in this series. Although diagnoses of carotid body tumors and adrenal pheochromocytomas in our cases have been made by clinical and laboratory findings, genetic screening should be performed for definitive diagnosis [15].

A ${ }^{123}$ I-MIBG scan is a sensitive diagnostic study to evaluate the presence of a previously undiagnosed synchronous paraganglioma, especially in patients with familial histories [10]. In this series, a preoperative ${ }^{131} \mathrm{I}$ MIBG scan revealed an uptake within the adrenal mass in 2 patients. The optimal management of carotid body tumors is still controversial. The treatment options are observation, surgical excision, embolism and radiotherapy. Factors influencing the choice of treatment include the patient's age, symptoms, size of the tumor, rate growth, presence of a glomus tumor elsewhere, and the side of the dominant hemisphere [6]. The occasional elderly patient with an asymptomatic tumor and a limited life expectancy is best treated by close follow-up. It is generally accepted that total surgical excision is a good treatment choice for carotid body tumors [16]. As such, surgical treatment was preferred to long follow-up time for the young or middle age patients. The current operative procedure has been described by Lees et al. [17] and Shamblin et al. [5]. Expert technique in catheter manipulation and embolic material injection is mandatory to avoid particle reflux into the cerebral or ophthalmic microvasculature. Therapeutic embolization is the other treatment option [18], successfully applied preoperatively in 3 patients in this study. However, Myers and Johnson [19] do not recommend preoperative embolization of carotid body tumors because these lesions can be dissected free of carotid artery without major blood loss. Additionally, they have observed that preoperative embolization may induce an intense inflammatory response that may in turn make subadventitial dissection of the tumor risky or even dangerous, resulting in intraoperative rupture of the carotid artery.

Radiation therapy (RT) should sometimes be considered as an effective treatment of paragangliomas. Conventional irradiation, laser or gamma knife irradiation is most often used in tumors with extensive intracranial or skull base involvement, multiple or bilateral tumors with potential postoperative debility from cranial nerve dys-

Med Princ Pract 2007;16:209-214 
function, and poor risk patients or when the patient withholds consent for surgery [14]. RT has been reported to provide excellent control for unresectable lesions and to be an adjunct to operation for incompletely excised tumors or metastases. Furthermore, there is no advantage of subtotal resection along with radiotherapy compared with radiotherapy alone. Therefore, the decision to use radiotherapy or surgery is based primarily on the risk of treatment complications [20]. In this report, no patient underwent RT.

\section{Conclusion}

Our data indicate that for early-stage jugulotympanic paraganglioma, carotid body paraganglioma and VP with a low risk of surgical complications, primary resection for cure is recommended. For larger tumors, numerous surgical series have shown excessive morbidity to cranial nerves and vascular structures if resection is attempted; therefore, we suggest radiotherapy alone under these conditions.

\section{References}

1 Williams PL, Warwick R (eds): Gray's Anatomy, ed 36. New York, Churchill Livingstone, 1980, pp 1462-1464.

- 2 Lack F, Cubilla A, Woodruff J, Farr HW: Paragangliomas of the head and neck region. A clinical study of 69 patients. Cancer 1977; 39:397-409.

3 Gulya AJ: The glomus tumor and its biology. Laryngoscope 1993;103:7-15.

4 McCaffrey TV, Meyer FB, Michels VV, Piepgras DG, Marion MS: Familial paragangliomas of the head and neck. Arch Otolaryngol Head Neck Surg 1994;120:1211-1216.

5 Shamblin WR, ReMine WH, Sheps SG: Carotid body tumor (chemodectoma): clinicopathologic analysis of ninety cases. Am J Surg 1971;122:732-739.

6 Sobol SM, Bailey JC: Familial multiple cervical paragangliomas: report of a hundred and review of the literature. Otolaryngol Head Neck Surg 1990;102:382-390.

7 Kaman L, Singh R, Aggarwal R, Kumar R, Behera A, Katariya RN: Diagnostic and therapeutic approaches to carotid body tumors: report of three cases and review of the literature. Aust N Z J Surg 1999;69:852-855.

${ }_{8}$ Thabet MH, Kotob H: Cervical paragangliomas: diagnosis, management and complications. J Laryngol Otol 2001;115:467-474.
9 Wax MK, Briant TD: Carotid body tumors: a review. J Otolaryngol 1992;4:277-285.

10 Chatal JF, Charbonnel B: Comparison of iodobenzylguanidine imaging with computed tomography in locating pheochromocytoma. J Clin Endocrinol Metab 1985;61:796872.

11 Persky MS, Setton A, Niimi Y, Hartman J, Frank D, Bernstein A: Combined endovascular and surgical treatment of head and neck paragangliomas - a team approach. Head Neck 2002;24:423-431.

12 Anand VK, Alemar GO, Sanders TS: Management of the internal carotid artery during carotid body tumor surgery. Laryngoscope 1995; 105:231-235.

13 Baysal BE, Myers EN: Etiopathogenesis and clinical presentation of carotid body tumors. Microsc Res Tech 2002;59:256-261.

14 Erickson D, Kudva YC, Ebersold MJ, Thompson GB, Grant CS, van Heerden JA, Young WF Jr: Benign paragangliomas: clinical presentation and treatment outcomes in $236 \mathrm{pa}$ tients. J Clin Endocrinol Metab 2001;86: 5210-5216.
15 Neumann HP, Bausch B, McWhinney SR, Bender BU, Gimm O, Franke G, Schipper J, Klisch J, Altehoefer C, Zerres K, Januszewicz A, Eng C, Smith WM, Munk R, Manz T, Glaesker S, Apel TW, Treier M, Reineke M, Walz MK, Hoang-Vu C, Brauckhoff $\mathrm{M}$, Klein-Franke A, Klose P, Schmidt H, MaierWoelfle M, Peczkowska M, Szmigielski C, Eng C, Freiburg-Warsaw-Columbus Pheochromocytoma Study Group: Germ-line mutations in nonsyndromic pheochromocytoma. N Engl J Med 2002;346:1459-1466.

16 Patetsios P, Gable DR, Garrett WV, Lamont JP, Kuhn JA, Shutze WP, Kourlis H, Grimsley B, Pearl GJ, Smith BL, Talkington CM, Thompson JE: Management of carotid body paragangliomas and review of a 30-year experience. Ann Vasc Surg 2002;16:331-338.

$\checkmark 17$ Lees CD, Levine HL, Beven EG, Tucker HM: Tumors of the carotid body. Experience with 41 operative cases. Am J Surg 1981;142:362365.

18 Dimakakos PB, Kotsis TE: Carotid body paraganglioma: review and surgical management. Eur J Plast Surg 2001;24:58-65.

19 Myers EN, Johnson JT: Neoplasms; in Cummings CW, Fredricson JM, Harker LA, Krause CJ, Richardson MA, Schuller DE (eds): Otolaryngology - Head and Neck Surgery, ed 2. St Louis, Mosby-Year Book, 1993, pp 1590-1604. 